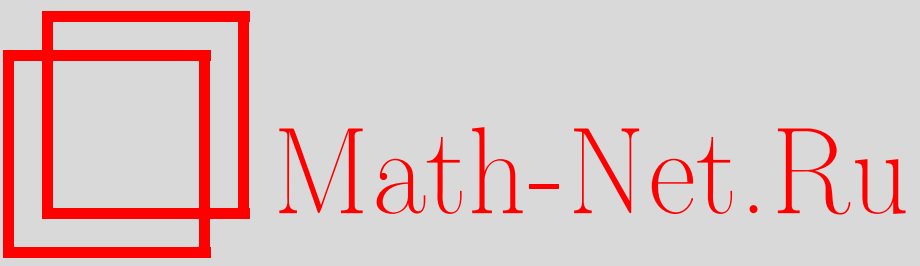

С. А. Довбыш, Трансверсальное пересечение сепаратрис, структура множества квазислучайных движений и несуществование аналитического интеграла в многомерных системах, УМН, 1996, том 51, выпуск 4, 153-154

DOI: https://doi.org/10.4213/rm1000

Использование Общероссийского математического портала Math-Net.Ru подразумевает, что вы прочитали и согласны с пользовательским соглашением

http://www.mathnet.ru/rus/agreement

Параметры загрузки:

IP : 3.80 .181 .102

26 апреля 2023 г., 03:27:11 


\title{
ТРАНСВЕРСАЛЬНОЕ ПЕРЕСЕЧЕНИЕ СЕПАРАТРИС, СТРУКТУРА МНОЖЕСТВА КВАЗИСЛУЧАЙНЫХ ДВИЖЕНИЙ И НЕСУЩЕСТВОВАНИЕ АНАЛИТИЧЕСКОГО ИНТЕГРАЛА В МНОГОМЕРНЫХ СИСТЕМАХ
}

\author{
С. А. Довьыш
}

1. Пусть $q$ - гиперболическая неподвижная точка $C^{N}$-диффеоморфизма $S n$-мерного многообразия $M$ на себя; $W^{-}$и $W^{+}$- ее выходящее и входящее инвариантные многообразия (сепаратрисы). Пусть размерности $W^{ \pm}$есть, соответственно, $n^{ \pm}$(тогда $n^{+}+n^{-}=n$ ) и $r_{j}$ - некоторые трансверсальные гомоклинические точки, так что в каждой точке $r_{j}$ многообразия $W^{-}$и $W^{+}$пересекаются трансверсально. Говорят, что орбиты точек $q, r_{j}$ образуют гомоклиническую структуру. Согласно теореме В.М. Алексеева [1], для любого открытого множества $U$, содержащего гомоклиническую структуру, существует открытое множество $V \subset U$ такое, что ограничение $S \mid A$ на максимальное $S$-инвариантное множество $A$, содержащееся в $V$, допускает описание методами символической динамики, т.е. топологически эквивалентно некоторой топологической марковской цепи (ТМЦ). Движения на множестве $A$ носят название квазислучайных, поскольку топологическая энтропия соответствующей ТМЦ является положительной [1], [2].

Хорошо известно, что в двумерном случае $\left(n=2, n^{ \pm}=1\right)$ множество $A$ квазислучайных движений является ключевым в смысле [3] для класса функций $F$, аналитических в окрестности $A \subset M$ (т.е. из $F \mid A \equiv 0$ следует $F \equiv 0$ ) и отсюда вытекает несуществование непостоянного аналитического (первого) интеграла диффеоморфизма $S$, т.е. функции $F$ на $M$ такой, что $F \circ S=F$. Соответствуюшее доказательство В.М. Алексеева [4] справедливо только для двумерного случая, однако, оно легко переносится на многомерный случай, если имеет место импликация

$$
F \in C^{N}(M), F|A \equiv 0 \Rightarrow d F| A \equiv 0,
$$

приводящая к ключевому свойству множества $A$ (в двумерном случае доказательство [4] основано на справедливости (1) при $N=1$ ). Более того, (1) влечет за собой несуществование нетривиальных мероморфного первого интеграла и аналитической однопараметрической группы симметрий [5] (или, более общо, мероморфного векторного поля, порождающего локальный фазовый поток, коммутируюший с отображением $S$ ). Условие (1) означает, что множество $A$ является "достаточно шероховатым" и не лежит ни на каком регулярном $C^{N}$-подмногообразии положительной коразмерности и даже на объединении счетного набора таких подмногообразий (последнее доказывается применением диагонального процесса). Подчеркнем, что не делается предположений о гамильтоновом происхождении диффеоморфизма $S$, а неинтегрируемость понимается в наиболее сильном аналитическом смысле.

Сфформулированные ниже достаточные условия, влекущие за собой (1), конструктивно проверяемы для конкретных динамических систем и сохраняются при малых возмущениях. В частности, в пространстве $C^{\infty}$-диффеоморфизмов, имеющих гомоклиническую траекторию, существует открытое всюду плотное подмножество неинтегрируемых.

2. Пусть $\lambda_{i}\left(1 \leqslant i \leqslant n^{+}\right), \mu_{i}\left(1 \leqslant i \leqslant n^{-}\right)$- все собственные числа отображения $S$ в точке $q$, причем $0<\left|\lambda_{i}\right|<1<\left|\mu_{i}\right|$. Предположим, что числа $\lambda_{i}$ удовлетворяют мультипликативным условиям нерезонансности

$$
\left|\lambda_{s}\right| \neq \prod_{i}\left|\lambda_{i}^{m_{i}}\right|
$$

для всех индексов $s$ и $m_{i} \in \mathbb{Z}^{+}$таких, что $\sum_{i} m_{i} \geqslant 2$. Введем отношение эквивалентности: $\lambda_{i^{\prime}} \sim \lambda_{i^{\prime \prime}}$, если и только если во всех неравенствах (2) знаки сохраняются при одновременной замене в левой и правой частях всех сомножителей $\lambda_{i^{\prime}}$ на $\lambda_{i^{\prime \prime}}$ или всех сомножителей $\lambda_{i^{\prime \prime}}$ на $\lambda_{i^{\prime}}$. 
Пусть $\left\{\Lambda_{1}^{+}, \ldots, \Lambda_{p^{+}}^{+}\right\}$- соответствующее разбиение $\left\{\lambda_{i}\right\}$ на классы эквивалентности, расположенные в порядке неубывания модулей. Аналогично, пусть $\left\{\mu_{i}\right\}$ также удовлетворяют мультипликативным условиям нерезонансности и $\left\{\Lambda_{1}^{-}, \ldots, \Lambda_{p^{-}}^{-}\right\}$- соответствующее разбиение $\left\{\mu_{i}\right\}$ на классы эквивалентности, расположенные в порядке невозрастания модулей. Целое число

$$
N>\max \left\{\ln \min _{i}\left|\lambda_{i}\right| / \ln \max _{i}\left|\lambda_{i}\right|, \ln \max _{i}\left|\mu_{i}\right| / \ln \min _{i}\left|\mu_{i}\right|\right\}
$$

будет ограничивать снизу гладкость диффеоморфизма $S$. Рассмотрим подпространства $L_{1}^{ \pm} \subset$ $\cdots \subset L_{p^{ \pm}}^{ \pm}=T_{q} W^{ \pm}$такие, что $L_{i}^{+}$(соответственно, $L_{i}^{-}$) отвечает жордановым клеткам для собственных чисел $\lambda_{s} \in \Lambda_{j}^{+}$(соответственно, $\mu_{s} \in \Lambda_{j}^{-}$), где $j \leqslant i$. По теореме С. Стернберга [6] существуют линеаризуюшие координаты $y^{ \pm} \in \mathbb{R}^{n^{ \pm}}$на $W^{ \pm}$, в которых отображение $S \mid W^{ \pm}$ принимает линейный вид $y^{ \pm} \mapsto J^{ \pm} y^{ \pm}$. Обозначим через $\mathfrak{N}^{ \pm} \subset \mathbb{R}^{n^{ \pm}}$множество $y^{ \pm}$-координат точек $r_{j} \in W^{ \pm}$и через $\widetilde{L}_{i, j}^{ \pm} \subset T_{r_{j}} W^{ \pm}$- пространства, получающиеся параллельными переносами из $L_{i}^{ \pm}$в линеаризующей системе координат. Пусть при положительных итерациях касательного отображения $T S^{\mp 1}: T M \rightarrow T M$ каждое пространство $\widetilde{L}_{i, j}^{ \pm}$стремится к $L_{i}^{ \pm}$. Для каждого собственного числа $\nu$ отображения $J^{ \pm}=S \mid W^{ \pm}$, линейного в координатах $y^{ \pm}$, проекция $\pi_{\nu}: \mathbb{R}^{n^{ \pm}} \rightarrow \mathbb{R}^{n^{ \pm}} / \operatorname{im}\left(J^{ \pm}-\nu \cdot \mathrm{id}\right)=\operatorname{coker}\left(J^{ \pm}-\nu \cdot \mathrm{id}\right)$ корректно определена, и пусть линейная оболочка множества $\pi_{\nu}\left(\mathfrak{N}^{ \pm}\right)$совпадает с $\pi_{\nu}\left(\mathbb{R}^{n^{ \pm}}\right)$.

ТеОРемА. При выполнении сформулированных выше условий справедлива импликаиия (1).

\section{СПИСОК ЛИТЕРАТУРЫ}

[1] Алексеев В. М. // УМН. 1981. Т. 36. №4. С. 161-176. [2] Алексеев В. М., Якобсон М. В. // Боуэн Р. Методы символической динамики. М.: Мир, 1979. С. 196-240. [3] Козлов В. В. // УМН. 1983. Т. 38. №1. С. 3-67. [4] Алексеев В. М. // Матем. сб. 1969. Т. 78. №1. С. 3-50. [5] Козлов В. В. // Прикл. матем. и мех. 1988. Т. 52. № 4. С. 531-541. [6] Sternberg S. // Amer. J. Math. 1957. V. 79. №4. P. 809-824.

НИИ механики

Принято редколлегией

Московского государственного 25.06.1996

университета им. М. В. Ломоносова 